\title{
Role of magnetic resonance cholangiopancreatography in biliary disorders
}

\begin{abstract}
Magnetic resonance cholangiopancreatography or MRCP is a technique that uses a powerful magnetic field to evaluate the disorders of liver, gallbladder, bile ducts, pancreas and pancreatic duct. This technique was introduced in 1991 \& has evolved rapidly over last two decades as it is non-invasive, does not require anaesthesia or contrast enhancing material and is less operator dependent. Although at present endoscopic cholangiopancreatography (ERCP) is the gold standard in evaluating the pancreaticobiliary disorders but is invasive, operator dependent and results in complications such as cholangitis, pancreatitis, haemorrhage and perforation of duodenum which have limited its routine use and has made MRCP as a routine investigation in evaluating biliary tree disorders. Furthermore MRCP is superior to ERCP in diagnosing the biliary disorders in the settings where biliary anatomy is distorted due to previous pancreatic biliary surgeries. MRCP is a technique that uses heavily weighted T2 sequences which highly enhances static or slowly flowing bile against non-enhancing surrounding soft tissue which makes the evaluation of biliary tree more accurate and easy.

Keywords: magnetic resonance imaging, magnetic resonance cholangiopancreatography, biliary ducts, gallstone, cholangiocarcinoma, liver, gallbladder, cholangitis, pancreatitis, haemorrhage, perforation, duodenum, anatomy, technique, diagnosing
\end{abstract}

Volume 2 Issue 4 - 2017

Sneha Karwa, Vitthalrao Vikhe Patil

Department of Radiodiagnosis and Imaging, India

Correspondence: Sneha Karwa, Department of Radiodiagnosis and Imaging, Memorial Hospital and Medical College, Ahmednagar, Maharashtra, India, Tel +919890849900, Email drsaurabhsbhutada@gmail.com

Received: October 29, 2016 | Published: March 14, 2017
Abbreviations: ERCP, endoscopic cholangiopancreatography; MRCP, magnetic resonance cholangiopancreatography; US, ultrasonography; CT, computed tomography; PTC, percutaneous transhepatic cholangiography; haste, half fourier acquisition single-shot turbo spin echo; MRI, magnetic resonance imaging

\section{Introduction}

Obstructive jaundice or biliary tract disorders are often common complaint of patients, and the majority of these patients turned out to have cholelithiasis. Biliary disorders are more common in females than males. Correct methods to detect common bile duct and pancreatic disease in patients with obstructive jaundice are important for treating surgeon to carry out appropriate treatment. For this purpose surgeons prefer the diagnostic modality which is non invasive, safe and highly sensitive in diagnosing biliary disorder as the treatment approach varies highly depending on the cause of biliary obstruction.

Evaluation of suspected biliary obstruction has traditionally involved a variety of imaging modalities including Ultrasonography (US), Computed Tomography (CT), and invasive cholangiography that includes Endoscopic Retrograde Cholangio-Pancreaticography (ERCP) and Percutaneous Transhepatic Cholangiography (PTC). Currently the non-invasive diagnosis of bile duct obstruction mainly relies on US and CT; however the accuracy of these techniques is limited because of low sensitivity for the diagnosis of stones in common bile duct. When compared with US and CT, ERCP is more accurate but is invasive and operator dependant and is associated with $1-7 \%$ morbidity and $0.2 \%-1 \%$ mortality. ${ }^{1}$ Also ERCP may be technically difficult and even impossible where anatomical variants are encountered or where anatomy is distorted due to previous surgical procedure. ${ }^{2}$ This fails the ERCP to examine the biliary and pancreatic ducts, upstream of obstruction making it difficult to exclude synchronous lesions and to plan appropriate therapeutic intervention. MRCP has emerged as a potent non-invasive alternative approach to evaluate the pancreatico-biliary system. The lack of need for sedation, i.v. contrast and radiation exposure and the advantage of it being noninvasive, able to delineate lesions at all levels in addition to being highly sensitive has made MRCP an important alternative to ERCP. ${ }^{3}$ MRCP plays vital role in diagnosing biliary tract disorders as it is noninvasive, operator independent with diagnostic accuracy

\section{Background and objectives}

a. To evaluate spectrum of findings in cases of biliary disorders on MRCP.

b. To evaluate role of MRCP in surgical management and outcome of biliary disorders.

\section{Materials and methods}

Study consists of fifty unselected patients of different age groups in whom there was clinical suspicion of biliary disease. This is a prospective cross sectional study carried out in department of radio diagnosis, DVVPF'S Medical College and Hospital, Ahmednagar. All cases of biliary pathology attending DVVPF'S Hospital, Ahmednagar were included in study, excluding those with cardiac pacemakers, prosthetic heart valves, cochlear implant or any metallic implant.

\section{Technique of MRCP}

The MRCP technique is performed with heavily T2-weighted turbo spin echo sequences and fast gradient echo sequences in which stationary fluid has resultant high signal intensity. ${ }^{4}$ It takes advantage 
of high signal intensity of body fluids on heavily T2W MRI. Static or slow moving fluid filled structures such as bile duct appears hyper intense areas, whereas background tissues some signal. This inherent difference in signal intensity enables MRCP to be carried out without contrast. ${ }^{5,6}$ MRCP is usually performed with heavily T2W sequences by using fat spin echo or SSFSE (Single Shot Fast Spin Echo) technique and both a thick collimation and thin collimation multi section technique with a torso phased array coil. The coronal plane is used to provide a cholangiographic display and the axial plane is used to evaluate $\mathrm{CBD}$.

For MRCP patients should be nil by mouth for 3-4 hours prior to the procedure. This reduces fluid content within the stomach, decrease duodenal peristalsis and promote the filling of gall bladder. MRCP is done by using 2 sequences - breath-hold and non-breathhold sequences. The breath-hold sequence acquires a single slab of data, between 40 and $80 \mathrm{~mm}$ thick, in 1 or 2 seconds. Thin slabs ( $4 \mathrm{~mm}$ thick) can also be acquired using breath-hold T2-weighted half Fourier acquisition single-shot turbo spin echo (HASTE) sequences. These are obtained in coronal or oblique coronal views. In addition, the MRCP involves acquiring multiple thin collimation slices, a nonbreath-hold, respiratory-triggered 3D turbo spin-echo (TSE) T2weighted sequence, $(1.5 \mathrm{~mm})$ that can be post-processed on an imaging workstation. The source images from a thin collimation multislice acquisition are reviewed in addition to the reconstructed images, in order to demonstrate small stones or other intraductal pathology that may be obscured by reconstruction effects.

\section{Results}

In the present study the cases of duct calculi predominated and was seen in 16 patients (32\%) fallowed by congenital (choledochal cyst) in $12(24 \%)$ and gall bladder masses in $6(12 \%)$.In our study, patients of biliary pathology especially stricture and mass lesion in lower part of CBD were better evaluated by MRCP. In patient with Klatskin tumour, in which hepatic ducts were better evaluated by MRCP (Table 1-3) (Figure 1-7).

Table I Sexwise distribution in the biliary diseases

\begin{tabular}{lll}
\hline Sex & No. of cases & percentage\% \\
\hline Males & 17 & $34 \%$ \\
Females & 33 & $66 \%$ \\
Total & 50 & 100 \\
\hline
\end{tabular}

Table 2 Age wise distribution in biliary diseases

\begin{tabular}{lll}
\hline Age (years) & No. of patients & Percentage (\%) \\
\hline $0-18$ & 3 & 6 \\
$19-40$ & 16 & 32 \\
$>40$ & 32 & 62 \\
Total & 50 & $100 \%$ \\
\hline
\end{tabular}

ERCP, histopathological reports and post-operative findings were compared. MRCP was $98 \%$ accurate in diagnosing the diseases. False negative result in one patient was due to technical problem. In this patient MRCP diagnosis was mass lesion in $2^{\text {nd }}$ part of duodenum, but on operation it was pancreatic head carcinoma.
Table 3 Number of patients showing various diseases

\begin{tabular}{|c|c|c|}
\hline Diagnosis & $\begin{array}{l}\text { No. of } \\
\text { cases }\end{array}$ & $\begin{array}{l}\text { MRCP diagnosis accuracy } \\
\text { (based on final diagnosis of } \\
\text { ERCP, histology, operative } \\
\text { findings) }\end{array}$ \\
\hline A) Congenital & 12 & \\
\hline Choledochal cyst & 12 & $100 \%$ \\
\hline B) Duct Calculi & 16 & \\
\hline In lower end of CBD & 7 & $100 \%$ \\
\hline In mid part of CBD & 5 & $100 \%$ \\
\hline In CHD & 4 & $100 \%$ \\
\hline C) Stricture & 6 & \\
\hline Benign & 3 & $100 \%$ \\
\hline Malignant & 3 & $100 \%$ \\
\hline D) Mass lesion & 16 & \\
\hline Klastkin tumour & 4 & $100 \%$ \\
\hline Periampullary carcinoma & 6 & $84 \%$ \\
\hline GB mass & 6 & $100 \%$ \\
\hline Total & 50 & \\
\hline
\end{tabular}

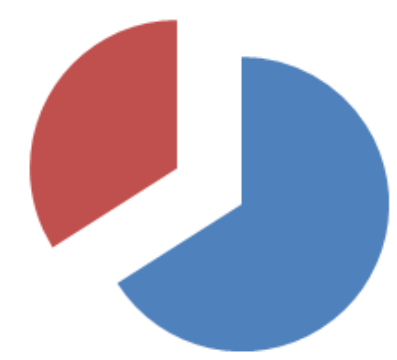

Figure I Females (66\%) V/S males (34\%).

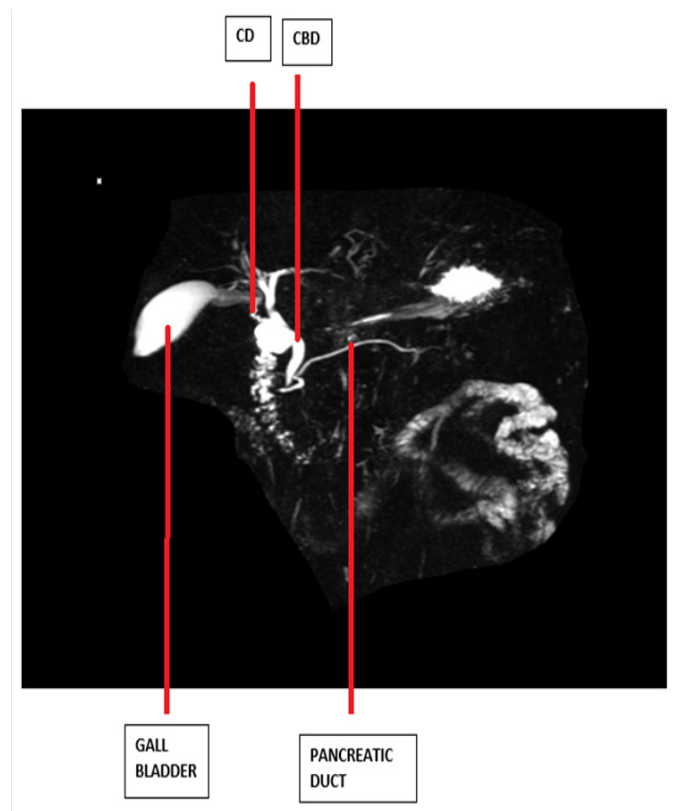

Figure 2 Normal anatomy of biliary system on MRCP.

$\mathrm{CD}$, cystic duct.

CBD, common bile duct. 


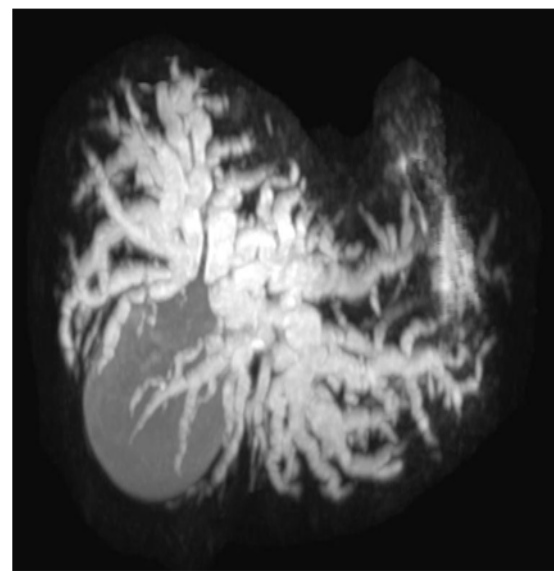

Figure 3 Hilar cholangiocarcinoma on MRCP.

Non visualisation of confluence of right and left hepatic ducts with dilated IHBR.

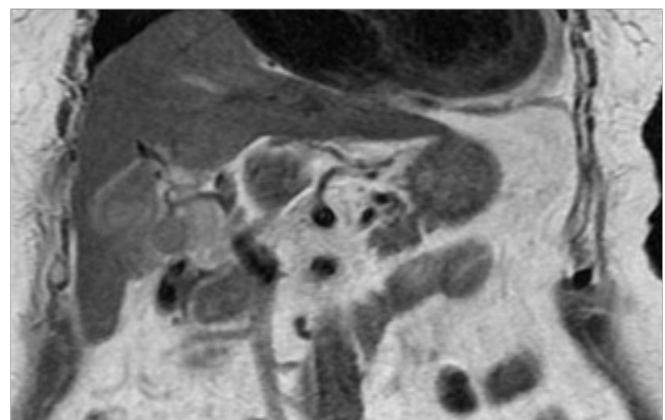

Figure 4A Gall bladder mass on TI W MRI.

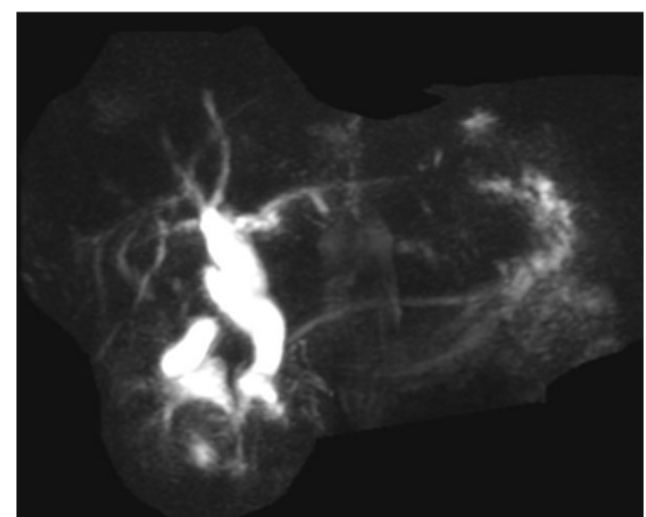

Figure 4B Gall bladder mass in same patient on MRCP.

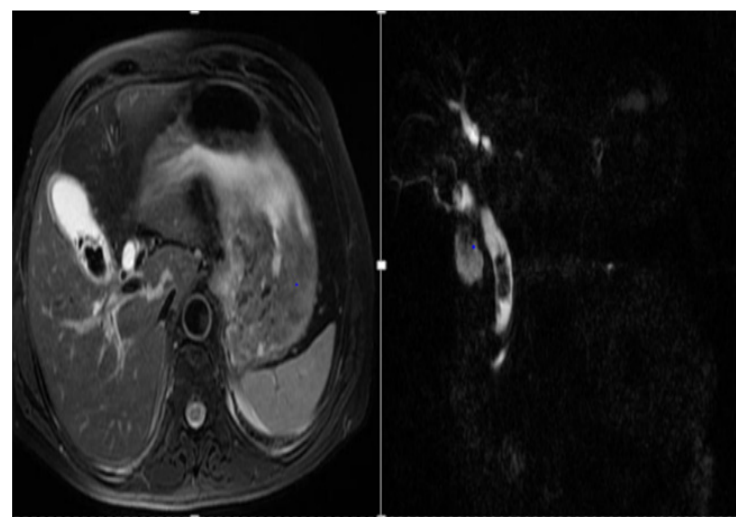

Figure 5A \& B Gall bladder and CBD caculi in patient on T2 W Mr Image (A) And Mrcp Image (B).

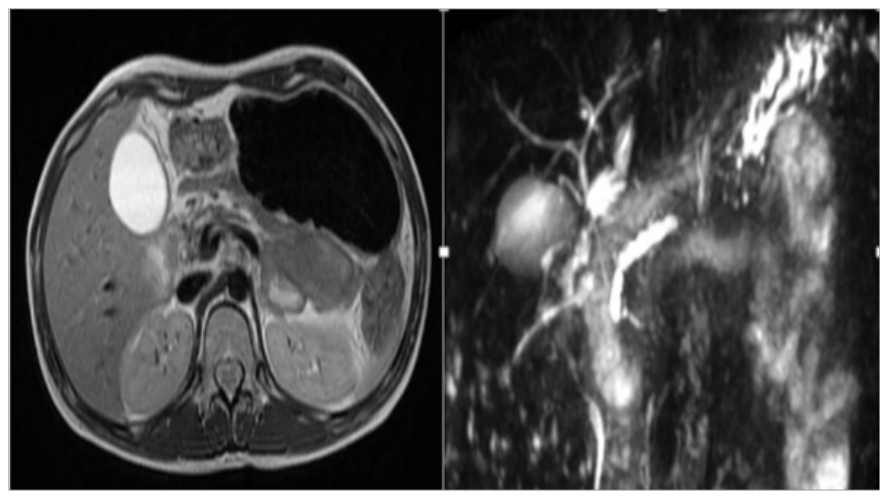

Figure 6A \& B Dialated pancreatic duct on T2W MR Image (A) AND MRCP Image (B).

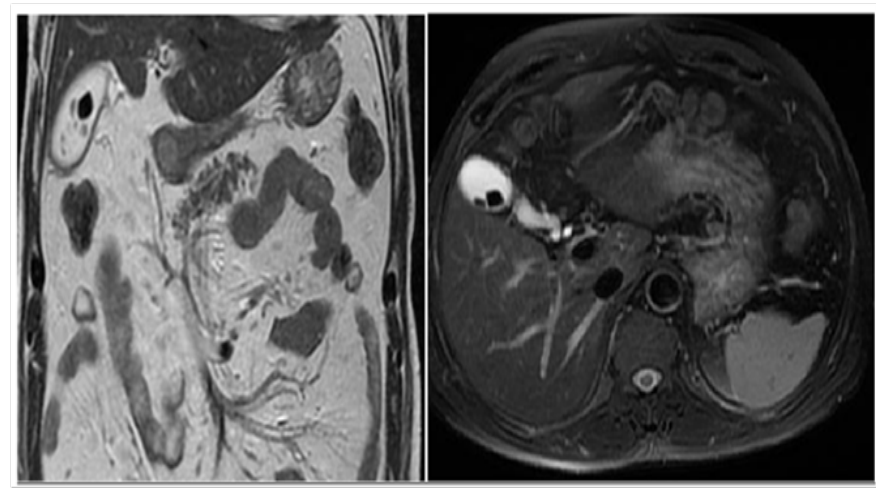

Figure 7A \& B Gall bladder stone on T2W MR Image (A) and MRCP Image (B).

\section{Discussion}

\section{Anatomy of biliary system}

Biliary tract includes right and left hepatic ducts, joins to form common hepatic duct. Cystic duct from gall bladder joins with common hepatic duct to form common bile duct. Common bile ducts have supraduodenal, retroduodenal, pancreatic and intraduodenal segments. Sphincter of oddi typically encircles the terminal portions of biliary and pancreatic ducts and their common channel (Table 4).

\section{Cholelithiasis and choledocholethiasis}

Choledocholithiasis accounts for most cases of biliary obstruction. Patients with obesity, increasing age, hyperalimentation, rapid weight reduction, illeal disease are considered at high risk of choledocholethiasis. $70-80 \%$ of gall stones in western countries are cholesterol and the remainders are pigment stones. On MRCP, calculi appear as foci of no or low signal intensity irrespective of their composition. A combination of thick slab MRCP technique and thin section multislice images increases the sensitivity for detection of large as well as small (1-4mm) stones. MRCP has a sensitivity of $81 \%$ to $93 \%$ and specificity of $91 \%$ to $95 \%$ in the evaluation of common bile stones which is comparable to sensitivity and specificity of ERCP for the evaluation of common bile duct stones.

\section{Gall stones produce three patterns of shadowing}

i. The $1^{\text {st }}$ is a discrete shadow emanating from a solitary stone.

ii. The $2^{\text {nd }}$ pattern is confluent shadowing due to multiple small stones "gravel" that abuts each other in gall bladder. 
iii. The $3^{\text {rd }}$ pattern wall echo shadow (wes) complex which occurs when a contracted gall bladder is completely filled with stones.

\section{Choledochal cyst}

This is uncommon cause of obstructive jaundice. It is frequently seen in female and asian infants.

It presents with classical triad of pain jaundice and right upper quadrant mass.

\section{Classification of alsono- lei modified by Todani et al. ${ }^{8}$}

I. Type IA: Cystic dilatation of CBD.

a) IB: Focal segmented dilatation of distal CBD.

b) IC: Fusiform dilatation of both CHD and CBD.

Table 4 Common causes of biliary obstruction

\begin{tabular}{ll}
\hline $\begin{array}{l}\text { Intrahepatic } \\
\text { biliary obstruction }\end{array}$ & $\begin{array}{l}\text { Portahepatic } \\
\text { biliary obstruction }\end{array}$ \\
\hline $\begin{array}{l}\text { Primary sclerosing carcinoma } \\
\text { Space occupying lesions and }\end{array}$ & Cholangiocarcinoma \\
liver diseases & Primary sclerosing carcinoma \\
& Gall bladder carcinoma \\
& Metastatic diseases \\
& Strictures \\
\hline
\end{tabular}

\section{Carcinoma of the gall bladder}

Carcinoma of gall bladder is fifth most common malignancy of the gastrointestinal tract. Major risk factors for gall bladder carcinoma includes gall stones in $65-95 \%$ of cases history of chronic cholecystitis in $40-50 \%$ cases and an estimated $22 \%$ of patients with porcelain gall bladder will develop gall bladder carcinoma. Gall bladder carcinoma has a peak incidence in $6^{\text {th }}$ decade of life. ${ }^{9}$

Gall bladder carcinomas can manifest as a polypoid mass with an intraluminal component, a bulky exophytic mass, or a mass infiltrating liver parenchyma and occupying the gall bladder lumen. Biliary obstruction may result from direct extension of the tumour to the porta hepatic or from compression of the extrahepatic bile ducts by enlarged lymph nodes.

The primary tumour and its spread beyond gall bladder appears hyper intense on T2WI and hypo intense on T1WI when compared with liver parenchyma.

Dynamic MRI with spoiled gradient pulse sequence may differentiate benign from malignant bladder lesions. Malignant lesions demonstrate early and prolonged enhancement where as benign lesion show different pattern. Dynamic MRI can also be useful for differentiation of chronic cholecystitis from carcinoma and for evaluation of weather the tumour invades beyond serosa. ${ }^{10}$

\section{Cholangiocarcinoma}

Cholangiocarcinoma is most common tumour of bile duct. Cholangiocarcinoma is classified as 1. Intrahepatic (peripheral) tumours 2. Hilar lesions occurring just past the confluence of right
II. Type II: True diverticulum arising from CBD.

III. Type III: Also called as choledochocele with cystic dilatation involving the intraduodenal portion of CBD.

IV. Type IVA: Multiple intra and extrahepatic cysts.

V. Type IVB: Multiple extrahepatic cysts.

VI. Type VC: Single or multiple intrahepatic cysts (Caroli's disease).

MRCP is non invasive procedure and provides the best available projection image for revealing the extent of choledochal cyst in children and adults. The development of single shot fast spin echo (SSFE) sequence made it possible to evaluate biliary tree in infants and children those who are not able to hold the breath.

\begin{tabular}{ll}
$\begin{array}{l}\text { Suprapancreatic biliary } \\
\text { obstruction }\end{array}$ & Intrapancreatic sites \\
\hline Pancreatic carcinoma & Pancreatic carcinoma \\
Metastatic diseases & Pancreatitis \\
Pancreatitis & Choledocholithiasis \\
Strictures & Cholangiocarcinoma \\
Cholangiocarcinoma & Ampullary carcinoma \\
Choledocholithiasis & Duodenal carcinoma \\
\hline
\end{tabular}

and left hepatic ducts commonly referred as "Klastkin" tumours; and 3. Distal ductal tumours. ${ }^{9}$

Whenever a cholangiocarcinoma is suspected, the fallowing aspects should be assessed: biliary dilatation, level of obstruction, presence of mass, focal or diffuse thickening of bile duct walls, hepatic metastasis, lymph nodes, portal vein thrombosis and cholelithiasis. ${ }^{11}$ The accuracy of MRCP in diagnosing the presence of obstruction ranges from $91-100 \%$ whereas the level of obstruction can be correctly evaluated in $85-100 \%$ while the accuracy in the differentiation of bening and malignant obstruction has varied within 30-98\%. The use of combination of MRI and MRCP improves diagnostic accuracy.

\section{Conclusion}

Based on the results of our study we have concluded that,

a) The introduction of MRCP has revolutionised the technique of non invasive evaluation of the anatomy and pathology of biliary tree.

b) Patient of suspected biliary obstruction or cholangiopathy can undergo MRCP as it is non-invasive, non-operator dependant $\&$ free of complication with diagnostic accuracy comparable to ERCP in evaluation of Biliary system.

c) Successful outcome in diagnosing the biliary pathology on MRCP depends on knowledge of technique of MR imaging and careful evaluation of wall of duct, ductal lumen and surrounding soft tissue structures.

\section{Acknowledgements}

None. 


\section{Conflict of interest}

Author declares that there is no conflict of interest.

\section{References}

1. Reinhold C, TaorelP, Bret P, et al. Choledocholithiasis: Evaluation of MR Cholangiography for diagnosis. Radiology. 1998;209(2):435-442.

2. Philip W Bearcroft, David J Lomas. Magnetic resonance cholangiopancreatography. Gut. 1997;41:135-137.

3. Soto JA, Barish MA, Yucel EK, et al. Magnetic resonance cholangiography; comparison with endoscopic retrograde cholangiopancreatography. Gastroenterology. 1996;110(2):589-597.

4. Larena JA, Astigarraga E, Saralegui I, et al. MRCP evaluation in pancreatic duct Pathology. Br J Radiol. 1998;71(850):1100-1104.

5. Macdonald OA, Peduto AJ. Magnetic resonance imaging and diseases of liver and biliary tract. Part 2 Magnetic resonance cholangiography and angiography and conclusions. J Gastroenterol Hepatol. 2000;15(9):992-999.
6. Barish MA, Yucel EK, Ferrucci JT. Magnetic Resonance Cholangiopancreatography. The New England Journal of Medicine. 1999;341(4):258-264.

7. James E Pearls. Advanced MRI-From Head to Toe. 2002;3:211-246.

8. Todani T, Wantanble Y, Narsue M, et al. Congenital bile duct cysts, classification, operative procedures and review of 37 cases including cancer arising from choledochal cyst. Am J Surg. 1997;134(2):263-269.

9. David Simpson. Radiology of the pancreas, gallbladder and Biliary tract. The Radiologic Clinics Of North America. 2002;40(6):11.

10. Yoshimitsu K, Honda H, Kaneko K, et al. Dynamic MRI of the gallbladder lesions: Differentiation of benign from malignant lesions. J Magn Reson Img. 1997;7(4):696-701.

11. Bloom CM, Langer B, Wilson SR. Role of US in detection, characterisation and staging of cholangiocarcinoma. Radiographics. 1991;19(5):1199-1218 